University of Wollongong

Research Online

Faculty of Engineering - Papers (Archive)

Faculty of Engineering and Information

Sciences

$1-1-1997$

\title{
The microscopic nonequilibrium process in solids under transient heating
}

B L. Zhou

Academia Sinica, Shenyang

$\mathrm{GH}$. He

Academia Sinica, Shenyang

Y J. Gao

Academia Sinica, Shenyang

W L. Zhao

Academia Sinica, Shenyang,wzhao@uow.edu.au

J D. Guo

Academia Sinica, Shenyang

Follow this and additional works at: https://ro.uow.edu.au/engpapers

Part of the Engineering Commons

https://ro.uow.edu.au/engpapers/5207

\section{Recommended Citation}

Zhou, B L.; He, G H.; Gao, Y J.; Zhao, W L.; and Guo, J D.: The microscopic nonequilibrium process in solids under transient heating 1997, 481-492.

https://ro.uow.edu.au/engpapers/5207

Research Online is the open access institutional repository for the University of Wollongong. For further information contact the UOW Library: research-pubs@uow.edu.au 


\title{
The Microscopic Nonequilibrium Process in Solids under Transient Heating ${ }^{1}$
}

\author{
B. L. Zhou, ${ }^{2}{ }^{3}$ G. H. He, ${ }^{2}$ Y. J. Gao, ${ }^{2}$ W. L. Zhao, ${ }^{2}$ and J. D. Guo ${ }^{2}$
}

\begin{abstract}
$\Lambda$ briel review of studies on the dynamic process of thermal expansion since the eighties is given. A magnitude of dillerence of one to two orders between the results of theoretical calculations and experimental observations of the characteristic time is discussed. The nonequilibrium localized phonon gas in a "hot spot" is studied using the Boltzmamn equation. leading to a soliton-like solution. It gives the way to fill the gap of the above-mentioned diflerence between theory and experiment. The lattice relaxition of a laser rod is developed theoretically and verilied experimentally. Diflerent expansion processes of a high- $T_{\mathrm{c}}$ superconductor below and above $T_{\text {c }}$ imply the existence of some new features of excitons in the high- $T_{\text {c statc. }}$
\end{abstract}

KEY WORDS: high- $T_{6}$ superconductor: nonequilibrium process; thermal expansion: transient heating.

\section{INTRODUCTION}

The dynamic process of thermal expansion in thin foils has been studied both theoretically and experimentally at this institute since the early eighties [1-4]. But one finds that there is a difference of about one to two orders of magnitude between the calculated and the observed characteristic time of the dynamic process [3-7].

Since then, some theoretical and experimental studies have been made. Some further experiments, concerning the microscopic nonequilibrium process in solids under transient heating, either by laser or by ohmic pulse, have also been performed. In the meantime, the macroscopic theory about

\footnotetext{
'Keynote lecture presented atl the Fourth Astim Thermophysical Properties Conference. September 5 8. 1995. Tokyo, Japan.

2International Center lor Materials Physics. Institute of Metal Research. Academia Sinica, Shenyang 110015 , P.R. China.

${ }^{3}$ To whom correspondence should be addressed.
} 
dynamic thermal expansion has been given elsewhere [8]. The experimental studies show not only the universality of the dynamic process of thermal expansion in solids, but also the possibility of improvement of material structures and properties by taking advantage of this dynamic effect.

\section{A BRIEF REVIEW OF STUDIES ON THE DYNAMIC PROCESS OF THERMAL EXPANSION}

The anharmonic vibration of a one-dimensional lattice can be described as [1]:

$$
m \frac{d u_{n}^{2}}{d t^{2}}=2 c\left[\left(u_{n+1}-u_{n}\right)-\left(u_{n}-u_{n} \quad 1\right)\right]-3 g\left[\left(u_{n+1}-u_{n}\right)^{2}-\left(u_{n}-u_{n} 1^{2}\right]\right.
$$

where $m$ is the mass of the particle, $t$ is time, $u_{n}$ is the displacement of the $n$th particle, $c$ is the stiffness of the first order, and $g$ is the stiffness of the second order.

By analytical treatment, the exact solution of this nonlinear differential equation set is [1]

$u_{n}(t)=p\left[i\left(\frac{18 g u_{0}}{m}\right)^{12}\left(\frac{T}{T_{01}}\right)^{10} a^{3} 2 t+\phi\right] u_{0}\left(\frac{T}{T_{0}}\right)^{13}\left(n^{3}+\frac{n}{2}\right) a^{3}+\frac{n c}{3 g}$

where $a$ is the lattice constant, $T_{11}$ and $T$ are temperatures, $u_{0}$ and $\phi$ are constants, and $p[\lambda t+\phi]$ is the Weierstrass elliptical function.

Taking statistical average. this solution yields [1]

$$
\tau_{N}=\frac{3 N g}{4 c^{2}} \sqrt{\frac{\pi m k T}{2}}
$$

where $k$ is the Boltzmann constant. Equation (3) implies that the characteristic time $\tau_{x}$ for the thermal expansion of the whole lattice is proportional to the length of the $N$-particle lattice.

The theoretical prediction of the dynamic process of thermal expansion had been verified by indirect observation through the electrical resistance measurement of a laser pulse-irradiated thin foil [4] and direct observations $[5,6]$. An infrared radiation pyrometer for transient temperature measurement and a photoelectric instrument for micrometric length-change measurement were developed. After being heated by a $\mathrm{CO}_{2}$ laser pulse $0.1 \mu \mathrm{s}$ in duration, the transient temperature-rise history and length change due to the thermal expansion of 20 to $50 \mu \mathrm{m}$-thick aluminum films over a 


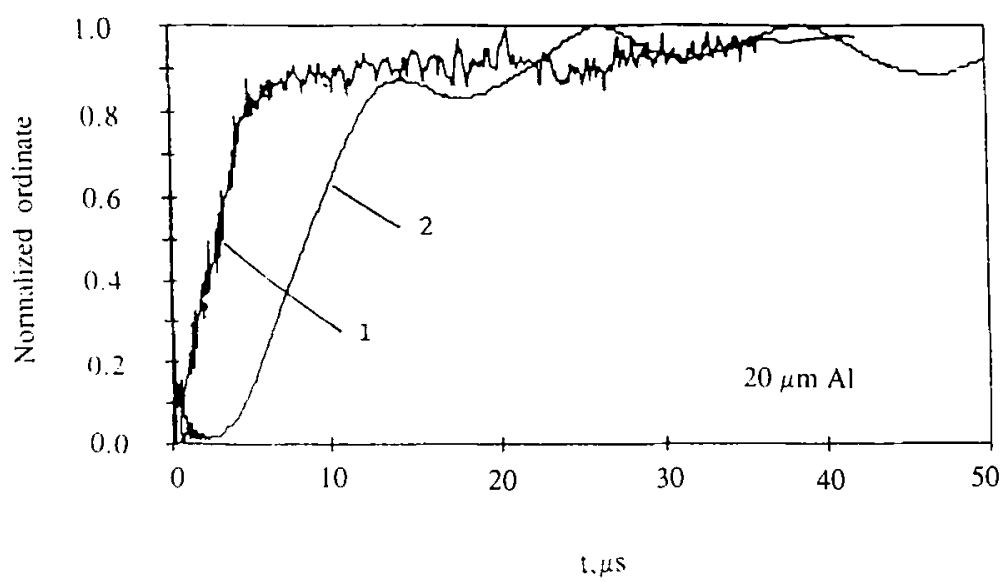

Fig. 1. Comparison of temperature rise (curve 1) and thermal-expansion (curve 2) versus time for laser pulse-heated, $20-\mu \mathrm{m}$-thick aluminium film $[3,5]$.

period of tens of microseconds were recorded. Nonsynchronous change of temperature and thermal expansion under transient heating was observed as shown in Fig. 1. Results on both conductors and insulators showed reasonable values for the time dependence of the expansion process on sample length [2].

Once the incident photons interact with electrons, the electron-electron, electron-phonon, and phonon-phonon interactions take place in the irradiated thin foil. To have an insight into the microscopic physical mechanism of the dynamic process. these interactions have been studied analytically [7]. The damping rates of the phonons in the different processes $\Gamma_{p}^{1}(\omega)$, etc., are as follows:

$$
\begin{aligned}
& \Gamma_{\mu}^{1}(\omega)=4 \pi \int \frac{d \vec{k}}{(2 \pi)^{2}}\left|V_{\iota}\right|^{2} \delta\left(E_{k}-E_{k-4}-\omega_{\iota}\right)\left[f^{\prime \prime}\left(E_{k-4}\right)-f^{\prime \prime}\left(E_{k}\right)\right] \\
& \Gamma_{Q}^{\prime}(E)=4 \pi \int \frac{d \vec{q}}{(2 \pi)^{2}}\left|V_{\iota}\right|^{2} \delta\left(E_{k}-E_{k-q}-\omega_{q}\right)\left[1+N^{0}\left(\omega_{q}\right)-f^{\prime \prime}\left(E_{k-q}\right)\right] \\
& \Gamma_{Q}^{2}(E)=4 \pi \int \frac{d \vec{q}}{(2 \pi)^{2}}\left|V_{q}\right|^{2} \delta\left(E_{k+q}-E_{k}-\omega_{u}\right)\left[N^{(4}\left(\omega_{\iota}\right)-f^{\prime \prime}\left(E_{k+q}\right)\right] \\
& \Gamma_{P}^{2}(\omega)=2 \pi \sum_{j_{1} j_{2}} \int \frac{d \vec{q}_{1}}{(2 \pi)^{2}}\left|V_{3}\left(-\vec{q} j, \vec{q}_{1} j_{1}, \vec{q}_{2} j_{2}\right)\right|^{2} \delta\left(\omega_{j q}-\omega_{j_{1} q_{1}}-\omega_{j_{2} q_{2}}\right) \\
& \times\left[1+N_{j_{1}}^{0}\left(\omega_{1}\right)+N_{j_{2}}^{0}\left(\omega_{2}\right)\right]
\end{aligned}
$$


Table I. Calculated Results for the Characteristic Time of the Damping Rate and Lifetime of Different Phonon Processes

\begin{tabular}{|c|c|c|c|c|c|}
\hline Mode & $\Gamma_{r}^{1}$ & $\Gamma_{0}^{1}$ & $\Gamma_{0}^{2}$ & $\Gamma_{i=1}^{2}$ & $\Gamma_{1}^{2}$ \\
\hline $\begin{array}{l}\text { Decaly rate (s } 1 \text { ) } \\
\text { Lifetime (s) }\end{array}$ & $\begin{array}{l}1.20 \times 10^{111} \\
8.30 \times 10^{11}\end{array}$ & $\begin{array}{l}1.20 \times 10^{111} \\
8.30 \times 10^{11}\end{array}$ & $\begin{array}{l}1.76 \times 10^{17} \\
5.68 \times 10^{11}\end{array}$ & $\begin{array}{l}3.49 \times 10^{7} \\
2.87 \times 10^{8}\end{array}$ & $\begin{array}{l}4.40 \times 10^{10} \\
2.28 \times 10^{\circ}\end{array}$ \\
\hline
\end{tabular}

where $\mathbf{k}$ is the electron wave vector, $E$ is the electron energy, $f(E)$ is the distribution of electrons, $V_{4}$ is the matrix elements of electron-phonon interaction, $N(0)$ is the distribution function of phonons, and $V_{3}$ is the matrix element of three-phonon interaction. It can be seen that there is still a one to two orders of magnitude difference between the characteristic time

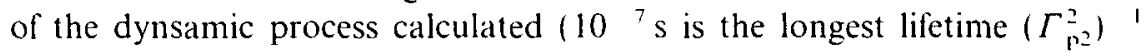
for the three-phonon process listed in Table I [3]) and that observed $\left(10 \cdots-10{ }^{5} \mathrm{~s}\right)$ [3]. In an attempt to explain this difference, both microscopic and macroscopic theoretical treatments have been made.

\section{NONEQUILIBRIUM LOCALIZED PHONON GAS IN A "HOT SPOT" [9]}

The nonequilibrium thermal process of localized phonons in a "hot spot", in which the phonon mean free path is greatly reduced by phononphonon scattering, can be described by the nonlinear Boltzmann equation [10]:

$$
\begin{aligned}
& {\left[\frac{\partial}{\partial t}+\vec{v}_{i} \cdot \nabla\right] n_{i}(\vec{k}, \vec{r} t)} \\
& \quad=-\left(4 \omega_{i}\right) \quad '\left[\prod _ { i j \cdot k } \left(\left(\omega_{i}, \vec{r} t\right) \cdot n_{i}(\vec{k}, \vec{r} t)-\prod_{i j} \sum_{k}\left(\left(w_{i}, \vec{r} t\right)\left(1+n_{i}(\vec{k}, \vec{r} t)\right)\right]\right.\right.
\end{aligned}
$$

where $n_{j}(\mathbf{k}, \mathbf{r} t)$ is the distribution function of phonons at position $\mathbf{r}$ and time $t$ with wave vector $\mathbf{k}, l_{\text {; }}$ is the phonon velocity, and $\omega_{j}$ is the phonon frequency. $\prod_{j i . \mathrm{k}}$ and $\prod_{j i . \mathrm{k}}$ are self-energy functions of phonons. If only the three-phonon process is considered, the right side of Eq. (8) can be expressed as [11]

$$
\begin{aligned}
-\frac{1}{2} h \pi & \sum_{i i_{1} j_{2}}\left|U_{i i_{1} j_{2}}^{\prime 3}\right|^{2}\left(2 \omega_{j} \cdot 2 \omega_{j_{1}} \cdot 2 \omega_{j_{2}}\right) \quad \cdot \cdot\left\{\delta ( \omega _ { j } - \omega _ { j _ { 1 } } - \omega _ { j _ { 2 } } ) \left[\left(1+n_{j_{1}}\right)\left(1+n_{j_{2}}\right) n_{j}\right.\right. \\
& \left.-n_{j_{1}} n_{j_{2}}\left(1+n_{j}\right)\right]+2 \delta\left(\omega_{i}-\omega_{j_{1}}+\omega_{j_{2}}\right) \\
& \left.\times\left[\left(1+n_{j_{1}}\right) n_{i_{2}} n_{j}-n_{j_{1}}\left(1+n_{j_{2}}\right)\left(1+n_{j}\right)\right]\right\}
\end{aligned}
$$


(a)

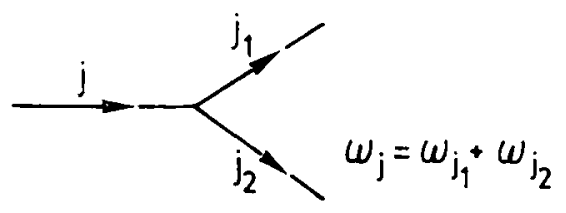

(b)

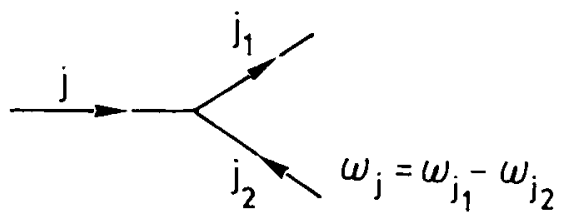

Fig. 2. Two catses of the three-phonon process.

where $U_{i i_{1}, j_{2}}^{\langle 3\rangle}$ is the nonlinear coefficient of lattice and $h$ is the Planck constant. There are two cases in the three-phonon process (Fig. 2).

For simplicity, Eq. (9) can be transformed into a one-dimensional form:

$$
\left(\frac{\partial}{\partial t}+v_{\mathrm{s}} \frac{\partial}{\partial x}\right) n_{j}=-a n_{j}^{2}-b n_{j}+b n_{i}^{\mathrm{E}}
$$

where $a=\pi h\left|U_{i i_{1}, j_{1}}^{(3)}\right|^{2}, b=\left(1 / \tau_{\mathrm{N}}+1 / \tau_{1}\right)$, and $\tau_{\mathrm{N}}$ and $\tau_{\mathrm{U}}$ are relaxation times [10] for normal and Umklapp processes, respectively, and $n_{i}^{F}=$ $1 /\left\{\exp \left[\beta\left(h \omega_{i}-r_{i}, P\right)\right]-1\right\}$ is the local equilibrium distribution function of phonons.

According to the Taniuti method [12], the solution of Eq. (10) is the expansion of steady and homogeneous solution $n_{i}^{\prime \prime}$ in the form of

$$
n_{j}=\bar{n}_{j}^{\prime}+\sum_{x=1}^{\prime} \varepsilon^{x} n_{i}^{(x)}(\ddot{k}, x t)
$$

where $\varepsilon$ is a small parameter and

$$
n_{i}^{(x)}(\vec{k}, x t)=\sum_{j}^{\prime} n_{i l}^{(x)}(\xi, \tau) e^{i(t k x-\cdots, n)}
$$

by definition $\xi=\varepsilon(x-v, t), \tau=\varepsilon^{2} t$, and $v_{s}=\partial \omega / \partial k$ is the group velocity of the second sound. Retaining the first major term and its conjugate term, the solution of Eq. (10) is then obtained:

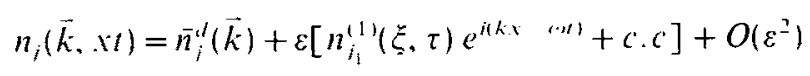


where $c . c$ is the conjugation symbol, and $n_{f_{1}}^{(1)}(\xi, \tau)=\phi^{(1)}(\xi, \tau)$. $n_{j-1}^{(1)}(\xi, \tau)=\left[\phi^{(1)}(\xi, \tau)\right]^{*}$, while $\phi^{(1)}(\xi, \tau)$ satisfies the following nonlinear Schrödinger equation:

$$
i \phi_{\tau}^{(1)}(\xi, \tau)+\beta \phi_{\xi \xi}^{\prime \prime \prime}(\xi, \tau)+\rho\left|\phi^{(1)}\right|^{2} \phi^{(1)}(\xi, \tau)=0
$$

where $\rho=-\left(n_{s}+b / 2 a\right)^{-1}, \beta=1 / 2\left(\partial \mathrm{v}_{\mathrm{s}} / \partial k\right)$, and let $\gamma^{\prime}$ be constant, thus the standard solution of Eq. (14) is

$$
\phi^{\prime \prime \prime}(\xi, \tau)=\left(\frac{-2 \gamma^{\prime}}{\rho}\right)^{12} \operatorname{sech}\left(\left(-\frac{\gamma}{\beta}\right)^{12} \xi\right) \exp \left(-i \gamma^{\prime} \tau\right)
$$

this is a wave-packet form soliton-like solution. The moving velocity $v_{\mathrm{s}}$ of the soliton-like wave-packet is about $10^{3} \mathrm{~m} \cdot \mathrm{s}{ }^{1}$ given in Ref. 13, and the lifetime of the long-lived tail of the phonons emitted from the "hot spot" will be $10 \mathrm{~mm} / 10^{3} \mathrm{~m} \cdot \mathrm{s}^{-1}=10 \mu$ for a $10-\mathrm{mm}$ sample. Consequently, the order of magnitude of the time calculated is in fairly good agreement with the experimental value [13].

The schematic illustration of the soliton-like wave-packet of phonon distribution in the "hot spot" is shown in Fig. 3a. Comparing it with the case of a laser pulse-irradiated thin foil of length $l=10 \mathrm{~mm}$, once the "hot spot" is formed at any point $x$ along the foil, the soliton-like wave-packet $\phi_{j-1}^{(1)}$, will move to the left and the other $\phi_{j_{1}}^{(1)}$ move to the right. Passing

(a)

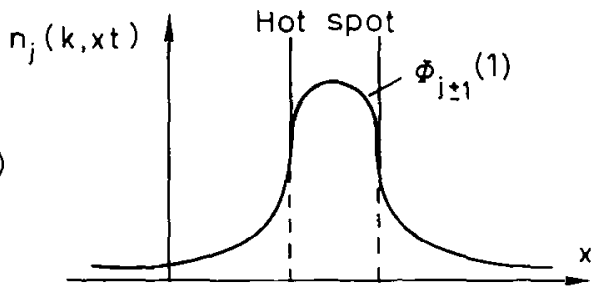

(b)

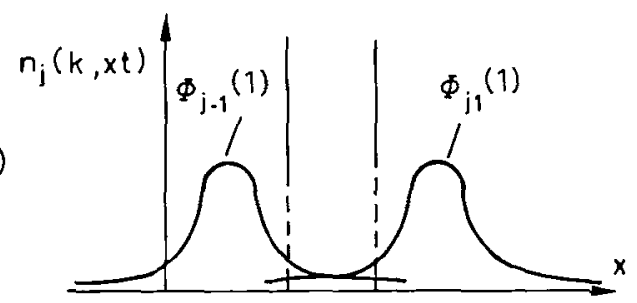

Fig. 3. (a) Phonon distribution in the "hot spot":

(b) extinction of the hot spot. 
through a path of length $x$ to a fixed point $x=0$, the packet $\phi_{j-1}^{(1)}$, will be reflected to the right along $l$ to the free end of the foil, while the other packet $\phi_{j_{1}}^{(1)}$ travels over $l-x$ to the free end (Fig. 3b). The total length traveled by the two wave-packets is $x+l+(l-x)=2 l$. Since the two packets starts their travel at the same time, the characteristic time will be $1 / l \int_{0}^{l}[(x+l / v-l-x / v)+l-x / v] d x=3 / 2 l / v=15 \mu \mathrm{s}$. This is also in fairly good agreement with the experimentally observed values [1-6], or in other words, the characteristic time for dynamic thermal expansion is proportional to the length of the sample and can be calculated quantitatively.

In addition, it is known from solid-state physics [14] that thermal expansion of a substance is proportional to the average thermal vibration energy in it, thus it can be said that the dynamic thermal expansion is also proportional to the phonon energy agitated by the laser pulse. Consequently, the time-dependent microscopic phonon distribution gives rise to the macroscopic time process for thermal expansion.

\section{LATTICE RELAXATION OF A LASER ROD}

All the samples studied in this laboratory on dynamic thermal expansion are opaque materials, which cannot be irradiated within the bulk. Next, we consider the case of a transparent material, such as the $\mathrm{Nd}$ glass rod in a laser equipment. The Hamiltonian can be written as

$$
H=H_{\mathrm{c}}+H_{\mathrm{cl}}+H_{\mathrm{l}}
$$

where $H_{c}, H_{c l}$, and $H_{1}$ are Hamiltonians of electrons, electron-phonon interaction, and the lattice, respectively.

The equilibrium position of any atom at $i$ electric state $Q_{\text {is }}$ is the sum of its equilibrium position in the ground state $Q_{s}$ and the lattice relaxation $\Delta_{\text {is }}[15,16]$. Next, the lattice relaxation and multiphonon nonradiative transition [17] are discussed. The transition matrix element is $[15,16]$

$$
\left\langle i^{\prime}|H| j n\right\rangle=\int \varphi_{i}(x, Q) x_{i n} \cdot(Q) H \varphi_{j}(x, Q) x_{j m}(Q) d x d Q
$$

By adiabatic approximation, it becomes

$$
\begin{aligned}
\left\langle\operatorname{in}^{\prime}|H| j n\right\rangle= & \int x_{i n^{\prime}}(Q)\left\{-\frac{h^{2}}{2} \sum_{s} \int \varphi_{i}(x, Q) \frac{\partial^{2}}{\partial Q_{s}^{2}} \varphi_{j}(x, Q) d x\right. \\
& \left.-h^{2} \sum_{s}\left[\int \varphi_{i}(x, Q) \frac{\partial}{\partial Q_{s}} \varphi_{j}(x, Q) d x\right] \frac{\partial}{\partial Q_{s}}\right\} x_{m}(Q) d Q
\end{aligned}
$$


while the nonradiative transition probability takes the form [15]

$$
W=\frac{2 \pi}{h} \mathrm{Av}_{n} \sum_{n^{\prime}}\left|\left\langle i n^{\prime}|H| j n\right\rangle\right|^{2} \delta\left(E_{i n^{\prime}}-E_{j n}\right)
$$

where $A v_{n}$ is the statistical average of the thermal distribution for different phonon states $n$ under certain electric states.

To generate a laser pulse, the $\mathrm{Nd}$ glass rod must be excited by $\mathrm{Xe}$ light. After the excitation but before the emission of the laser pulse, the multiphonon nonradiative transition plays an important role, and the lattice temperature goes up with the lattice expansion. Once the laser pulse emits, together with electron-level transitions, the lattice relaxation comes into action and causes a small amount of lattice contraction superposing on the normal dynamic thermal expansion. To check these assumptions,
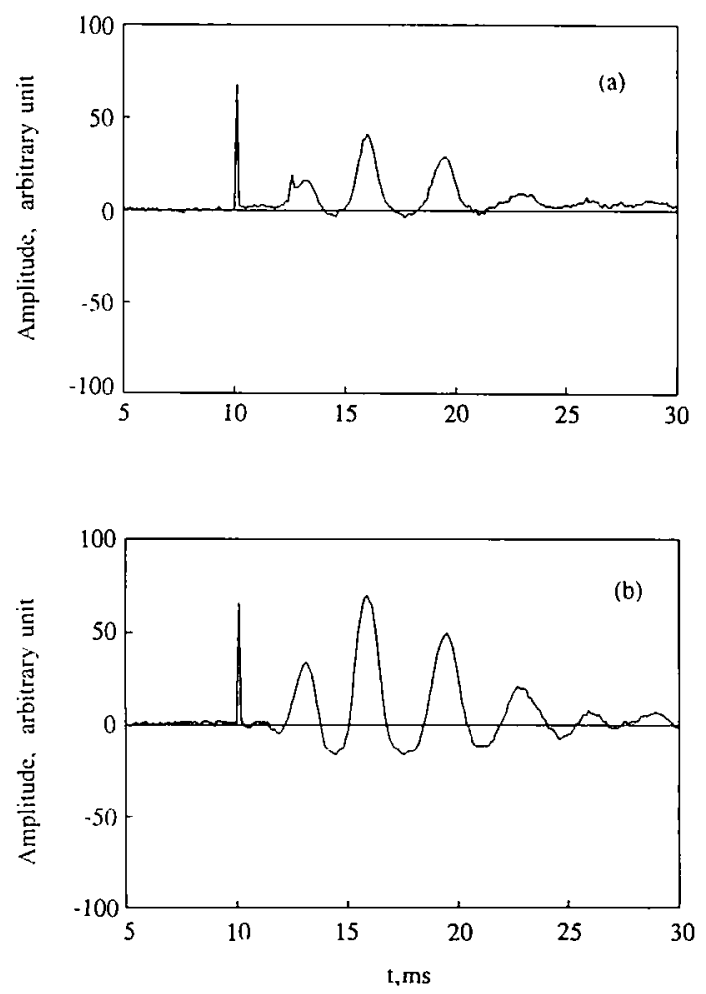

Fig. 4. Length change versus time for a Nd glass laser rod: (a) with laser pulse emission; (b) without laser pulse emission. 
some experiments were conducted [18]. The length change processes of the laser rod excited by Xe light, with and without laser pulse emission are shown in Fig. 4.

It can be seen from Fig. 4 that (a) the amplitude of the dynamic length change of the rod is much larger than that of the steady-state change, shown by the portion after oscillation-the dynamic length change will cause much stronger thermal stress than that predicted by the elastic theory; (b) the characteristic time of thermal expansion in the case with the laser pulse emission is about $200 \mu$ s longer than that without the emission; and (c) the amplitude of the former case is smaller than the latter.

It should be noted that the shape of the curve for a transparent material is much different from that for an opaque material.

\section{DIFFERENT EXPANSION PROCESS FOR A HIGH- $\boldsymbol{T}_{\mathrm{c}}$ SUPERCONDUCTOR BELOW AND ABOVE THE $T_{\mathrm{c}}$ TEMPERATURE [19]}

It is known that the steady-state thermal expansion of high- $T_{\mathrm{c}}$ superconducting materials makes almost no difference below and above the superconducting transition temperature $T_{c}$ [20] as shown in Fig. 5. One almost cannot find the evident transition point on the temperature-dependent curve of thermal expansion in the vicinity of the temperature range corresponding to the transition temperature $T_{\mathrm{c}}$ of electric conductivity.

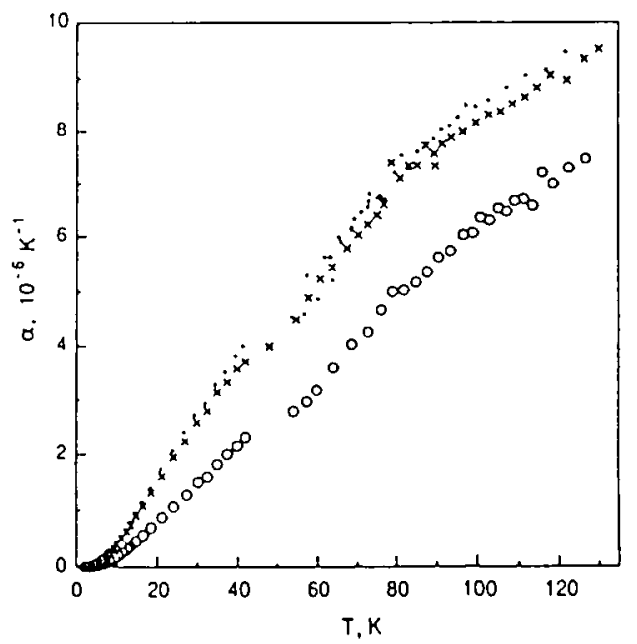

Fig. 5. $x(T)$ below $130 \mathrm{~K}$ for BPSC6 (- $)$ BSC2 $(x)$, and BPSC8 $(1,120]$. 
Some recent observations on the dynamic process of the thermal expansion of high- $T_{c}$ superconducting material $\mathrm{Bi}_{2} \mathrm{Sr}_{2} \mathrm{Ca}_{2} \mathrm{Cu}_{3} \mathrm{O}_{10}$ (BSCCO) thin foils have been made in this laboratory [19]. The BSCCO samples were prepared at this institute by the High- $T_{c}$ Group. The sample size is $25 \mathrm{~mm}$ in length, $5 \mathrm{~mm}$ in width, and $300 \mu \mathrm{m}$ in thickness, and the transition temperature $T_{\mathrm{c}}$ is $105 \mathrm{~K}$.

The dynamic process of thermal expansion of these samples was observed essentially in the same manner as before [2,5], but the lowest temperature was near $80 \mathrm{~K}$. Figure 6 shows the typical curves of length change versus time for the same sample under transient laser pulse $(0.1 \mu \mathrm{s})$ heating at different temperatures. It can be seen that for the same sample under same heating energy, the amplitude at $83 \mathrm{~K}\left(<T_{\mathrm{c}}\right)$ (Fig. 6a) is almost one order of magnitude higher than that at $113 \mathrm{~K}\left(>T_{\mathrm{c}}\right.$ ) (Fig. 6b). This phenomenon was repeatable many times [19].
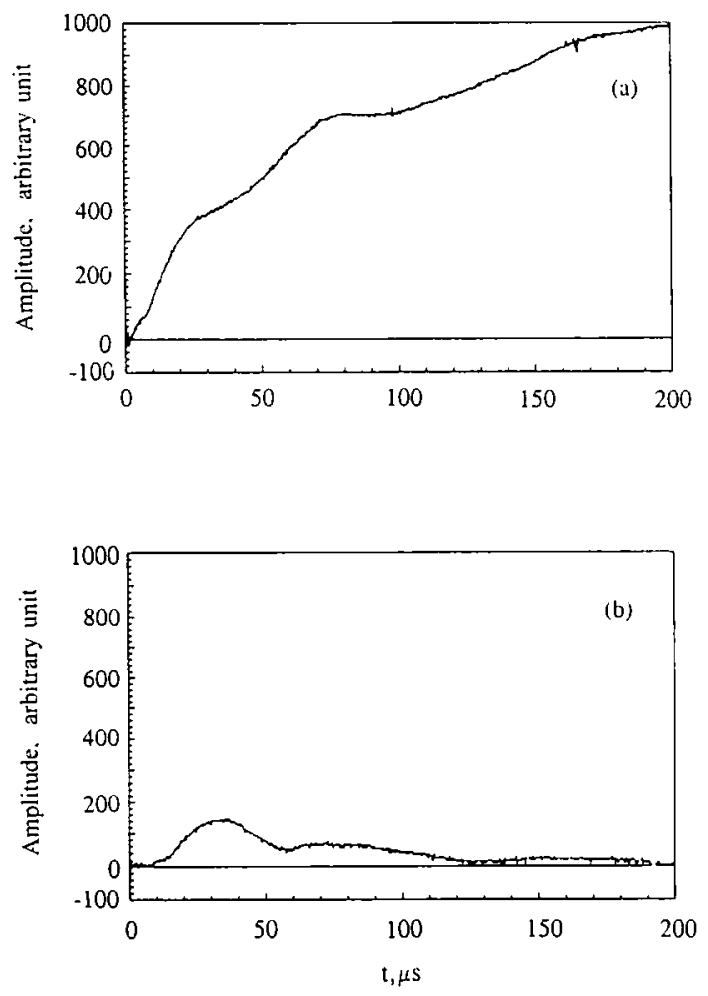

Fig. 6. Lenght change versus time for the same thin foil sample of a high- $T_{\text {c }}$ superconductor $\mathrm{Bi}_{2} \mathrm{Sr}_{2} \mathrm{Ca}_{2} \mathrm{Cu}_{3} \mathrm{O}_{111}$ under laser pulse heating at different temperatures: (a) at $83 \mathrm{~K}$ and (b) at $133 \mathrm{~K}\left(T_{\mathrm{c}}=105 \mathrm{~K}\right)$. 
It is believed that as long as BSCCO is in the normal state above $T_{\mathrm{c}}$, there are only a few free electrons participating in the interaction with incident photons together with some localized electrons. That is only a small amount of energy can be imparted to the lattice to cause expansion, while in the superconducting state below $T_{\mathrm{c}}$, a great number of carriers will be excited by the incident photons and a large amount of energy can be absorbed. The carriers are believed to be a new kind of excitons to be determined. Once the photon energy is brought in, the excitons are excited to a higher temperature than the lattice and then transfer their energy to the phonons through a series of particle-particle interactions as described in the preceding sections. Consequently, the amplitude of the length change of BSCCO below $T_{c}$ is much higher than that above $T_{c}$.

\section{CONCLUDING REMARK}

A series of theoretical and experimental studies on the dynamic process of thermal expansion of solids shows the universality of dynamic phenomenon, and the characteristic time of the expansion process calculated is in fairly good agreement with the observed result. The results on lattice relaxation of a laser rod and a high- $T_{\mathfrak{v}}$ superconductor are interesting and worthy of further studies.

\section{ACKNOWLEDGEMENTS}

The authors are grateful to Prof. Zhou Guangzhao, President of the Chinese Academy of Sciences, for his enthusiastic support and special fund and to Prof. Zhang Cunhao, Dalian Institute of Chemical Physics, Academia Sinica, for his generous support of the experimental facilities at the beginning of this work. The authors are also grateful to Prof. P. G. Klemens, University of Connecticut, Storrs, USA, for his valuable advice. The project was supported by the National Natural Science Foundation of China (5880245).

\section{REFERENCES}

I. B. L. Zhou, in Proc. Ist Asiun Therm. Prop. Conf. (ATPC) (China Academic Publishers, Beijing. 19861. p. 39.

2. B. L. Zhou. Proc. Ind ATPC (Salpporo, 1989), p. 17.

3. B. L. Zhou. Proc. 3rd ATPC (Beijing, 1992), p. I.

4. C. F. Xu, B. L. Zhou, and Q. H. Ye. Proc. Ist ATPC (Beijing, 1986), p. 587.

5. D. W. Tang. B. L. Zhou, H. Cao, and G. H. He, Appl. Phrs. Letr. 59:3113 (1991).

6. D. W. Tang. B. L. Zhou, H. Cao, and G. H. He. J. Appl. Phy's. 73:3749 (1993). 
7. J. X. Li and B. L. Zhou. J. Phis. Conklns. Mank' 4:1015 (1992).

8. D. W. Tang. N. Araki. N. Noda, and B. L. Zhou, J. Phyx. D Appl. Phas. 28:990 (1995).

9. X. S. Ling. Y. J. Galo, and B. L. Zhou, Proc. ylh Natl. Simp. Noneq. Statisl. Phys. (Nanning. China. 1994), p. 143 (in Chinese).

10. P. C. Kwok, Sulid Stanc Phis, 20:21311967).

11. I. Callaway, Quantam Theory of the Solid Statc (Acadenic Press. New York, 1976), p. 66 (Chinese ed.).

12. T. Taniuli and N. Yajima, J. Math. Phys. 10:1369 (1969).

13. M. Greenstein, M. A. Tamer, and J. P. Wolle, Phis. Re't. 1326:5604 (1982).

14. X. D. Xie and J. X. Fang. Solid Shate Ph!sics (Shanghai. 1962), p. 118 (in Chinese).

15. K. Huang. Prog. Phys, 1:31 (1981) (in Chinese).

16. K. Huang and A. Rinys, Proc: Roy. Soc. A204:406 (1950).

17. H. W. Moos, J. Luminess: 1-2:106 (1970).

18. Z. C. Yan. Master degree thesis (Inst. Met. Res.. Acad. Sinica, 1994).

19. J. D. Guo, Doctorate dissertation (Inst. Met. Res. Acad. Sinica, 1995).

20. (j. K. White, R. Driver, and R. B. Roberts, /m. I. Themophirs. 12:687 (1991). 